\title{
Article \\ Multifaceted Analysis of IL-23A- and/or EBI3-Including Cytokines Produced by Psoriatic Keratinocytes
}

\author{
Kota Tachibana ${ }^{1}{ }^{(D)}$, Nina Tang ${ }^{1,2}$, Hitoshi Urakami ${ }^{1}$, Ai Kajita ${ }^{1}$, Mina Kobashi ${ }^{1}$, Hayato Nomura ${ }^{1}$, \\ Minori Sasakura ${ }^{1}$, Satoru Sugihara ${ }^{1}$, Fan Jiang ${ }^{3}$, Nahoko Tomonobu ${ }^{3}$, Masakiyo Sakaguchi ${ }^{3}$ (D), \\ Mamoru Ouchida ${ }^{4}$ and Shin Morizane ${ }^{1, *(\mathbb{D}}$
}

check for updates

Citation: Tachibana, K.; Tang, N.; Urakami, H.; Kajita, A.; Kobashi, M.; Nomura, H.; Sasakura, M.; Sugihara, S.; Jiang, F.; Tomonobu, N.; et al. Multifaceted Analysis of IL-23Aand/or EBI3-Including Cytokines Produced by Psoriatic Keratinocytes. Int. J. Mol. Sci. 2021, 22, 12659. https://doi.org/10.3390/ ijms222312659

Academic Editor: Naoko Kanda

Received: 1 November 2021

Accepted: 20 November 2021

Published: 23 November 2021

Publisher's Note: MDPI stays neutral with regard to jurisdictional claims in published maps and institutional affiliations.

Copyright: (c) 2021 by the authors. Licensee MDPI, Basel, Switzerland. This article is an open access article distributed under the terms and conditions of the Creative Commons Attribution (CC BY) license (https:// creativecommons.org/licenses/by/ $4.0 /)$.
1 Department of Dermatology, Okayama University Graduate School of Medicine, Dentistry, and Pharmaceutical Science, 2-5-1 Shikata-cho, Kitaku, Okayama 700-8558, Japan; py4r2ift@okayama-u.ac.jp (K.T.); tanna@126.com (N.T.); p2ra59n8@s.okayama-u.ac.jp (H.U.); gmd421029@s.okayama-u.ac.jp (A.K.); mina584@okayama-u.ac.jp (M.K.); pnx69sky@okayama-u.ac.jp (H.N.); me422114@s.okayama-u.ac.jp (M.S.); pav10623@okayama-u.ac.jp (S.S.)

2 Department of Dermatology, Southern Medical University Zhujiang Hospital, 253 Gongye Middle Ave, Guangzhou 510280, China

3 Department of Cell Biology, Okayama University Graduate School of Medicine, Dentistry, and Pharmaceutical Science, 2-5-1 Shikata-cho, Kitaku, Okayama 700-8558, Japan;

pdbq7wx4@s.okayama-u.ac.jp (F.J.); n-tomonobu@okayama-u.ac.jp (N.T.); masa-s@md.okayama-u.ac.jp (M.S.)

4 Department of Molecular Oncology, Okayama University Graduate School of Medicine, Dentistry, and Pharmaceutical Science, 2-5-1 Shikata-cho, Kitaku, Okayama 700-8558, Japan; ouchidam@md.okayama-u.ac.jp

* Correspondence: zanemori@cc.okayama-u.ac.jp

Abstract: Interleukin (IL) 23 (p19/p40) plays a critical role in the pathogenesis of psoriasis and is upregulated in psoriasis skin lesions. In clinical practice, anti-IL-23Ap19 antibodies are highly effective against psoriasis. IL-39 (p19/ Epstein-Barr virus-induced (EBI) 3), a newly discovered cytokine in 2015, shares the p19 subunit with IL-23. Anti-IL-23Ap19 antibodies may bind to IL-39; also, the cytokine may contribute to the pathogenesis of psoriasis. To investigate IL23Ap19- and/or EBI3-including cytokines in psoriatic keratinocytes, we analyzed IL-23Ap19 and EBI3 expressions in psoriasis skin lesions, using immunohistochemistry and normal human epidermal keratinocytes (NHEKs) stimulated with inflammatory cytokines, using quantitative real-time polymerase chain reaction (RT-PCR), enzyme-linked immunosorbent assay (ELISA), and liquid chromatographyelectrospray tandem mass spectrometry (LC-Ms/Ms). Immunohistochemical analysis showed that IL-23Ap19 and EBI3 expressions were upregulated in the psoriasis skin lesions. In vitro, these expressions were synergistically induced by the triple combination of tumor necrosis factor (TNF)- $\alpha$, IL-17A, and interferon (IFN)- $\gamma$, and suppressed by dexamethasone, vitamin D3, and acitretin. In ELISA and LC-Ms/Ms analyses, keratinocyte-derived IL-23Ap19 and EBI3, but not heterodimeric forms, were detected with humanized anti-IL-23Ap19 monoclonal antibodies, tildrakizumab, and anti-EBI3 antibodies, respectively. Psoriatic keratinocytes may express IL-23Ap19 and EBI3 proteins in a monomer or homopolymer, such as homodimer or homotrimer.

Keywords: psoriasis vulgaris; interleukin (IL) 23; IL-39; p19; Epstein-Barr virus-induced (EBI) 3; tildrakizumab

\section{Introduction}

Psoriasis is a chronic inflammatory skin disease characterized by epidermal thickening and neutrophil and T-cell infiltration [1]. It is clinically characterized by well-dermacated, scaly erythematous plaques that typically develop on the scalp, elbows, knees, and buttocks [2]. Patients with psoriasis have inflammation not only on the skin, but also on the whole body, which increases the risk of various complications, such as psoriatic arthritis, cardiovascular disease, diabetes mellitus, obesity, and so on [3-8]. 
Interleukin (IL) 23 plays a critical role in the pathogenesis of psoriasis and is increased in the lesional skin of psoriasis [9-13]. IL-23 is a member of the IL-12 cytokine family forming heterodimers comprised of $\alpha$-subunits $\mathrm{p} 19$ and $\beta$-subunits $\mathrm{p} 40$ [14]. In addition to IL-23, the IL-12 cytokine family includes IL-12 (p35/p40), IL-27 (p28/EBI3), IL-35 (p35/EBI3), and IL-39 (p19/EBI3). IL-23 drives the differentiation, proliferation, and maintenance of T helper 17 (Th17) cells [14-16]. Anti-IL-23Ap19 antibodies (risankizumab, tildrakizumab, guselkumab) are already known to be clinically effective against moderateto-severe psoriasis [17-19]. On the other hand, p40, which constitutes IL-12 with p35 and IL-23 with p19, is also a therapeutic target for psoriasis. However, anti-IL-12/23p40 antibodies (ustekinumab) are less effective than anti-IL-23Ap19 antibodies for psoriasis [20]. Some groups reported that collateral targeting of IL-12 by anti-IL-12/23p40 antibodies is counterproductive in the therapy of psoriasis [21].

A cytokine composed of IL-23Ap19 and Epstein-Barr virus-induced (EBI) 3 heterodimer was found in transfected HEK293 cells by Ramnath et al. in 2015 [22]. The following year, the novel cytokine was named IL-39 (p19/EBI3) and was shown to be produced by $B$ cell lymphocytes and activated neutrophils $[23,24]$. IL-39 was shown to mediate inflammation in lupus-like mice and act as a signal in both the IL-23R/gp130 receptor and the signal transducer and activator of transcription (STAT) 1/STAT3 pathways [23]. AntiIL-39 polyclonal antibodies were shown to improve autoimmune symptoms in lupus-like mice [25]. In theory, the anti-IL-23Ap19 antibodies can bind to both IL-23 and IL-39, which suggests that both cytokines contribute to the pathogenesis of psoriasis, based on the clinical success of anti-IL23Ap19 antibodies against moderate-to-severe psoriasis. In addition, the existence of IL-39 in psoriatic skin might explain why anti-IL23Ap19 antibodies are more effective than anti-IL-12/23p40 antibodies. On the other hand, some reports have suggested no evidence of the existence or function of IL-39 in human and human immune cells [26,27]. Recent publications assessing the combinatorial potential of cytokine subunits in the IL-12 family failed to detect IL-39 in transfected HEK293 cells [28]. Expressions of IL23Ap19 and EBI3 mRNAs were detected by quantitative polymerase chain reaction (qPCR) in human epidermal keratinocytes stimulated with polycytidylic acid (Poly (I:C)) and in oral epithelial cells stimulated with IL-36 $\gamma[22,29]$. However, little information is available on the production of IL-23Ap19- and/or EBI3-including cytokines, such as IL-23 (p19/p40), IL-27 (p28/EBI3), IL-35 (p35/EBI3), and IL-39 (p19/EBI3), in human epidermal keratinocytes.

Further detailed analysis of the expressions of these cytokines in the cells and their involvement in the pathogenesis of psoriasis will contribute to the elucidation of the pathophysiology of psoriasis and may unlock new therapeutic options. Therefore, in this study, we investigated IL-23Ap19- and/or EBI3-including cytokines in psoriatic keratinocytes, using transcriptional and proteomic analysis.

\section{Results}

\subsection{The Expression of IL-23Ap19 and EBI3 Is Upregulated in Psoriatic Keratinocytes}

To investigate whether human epidermal keratinocytes express IL-23Ap19 and EBI3 in psoriasis, we first immunostained normal skin and lesional psoriatic skin sections with polyclonal rabbit anti-IL-23Ap19 antibody and monoclonal mouse anti-EBI3 antibody. As shown in Figure 1, immunohistochemical staining showed IL-23Ap19 and EBI3 expression in the epidermal keratinocytes. The expressions of IL-23Ap19 and EBI3 were intra- and perinuclear in normal skin. (Figure 1A,C). However, in psoriatic skin, IL-23Ap19 and EBI3 were expressed diffusely throughout the entire epidermis in addition to strong intra- and perinuclear staining (Figure 1B,D).

\subsection{TNF- $\alpha, I L-17 A$, and IFN- $\gamma$ Synergistically Induce IL-23Ap19 and EBI3 Expression in NHEKs}

Epidermal keratinocytes respond to TNF- $\alpha$, IL-17A, and IFN- $\gamma$, which are involved in the pathogenesis of psoriasis, in part by producing inflammatory cytokines, chemokines, 
and antimicrobial peptides [30,31]. Chiricozzi et al. showed that keratinocytes synergistically upregulated these expressions by the combination of TNF- $\alpha$ and IL-17 in the cells [30]. The detected genes from keratinocytes stimulated with TNF- $\alpha$ and IL-17 included some of the highest expressed genes in psoriatic skin. This indicated an impressive correlation between IL-17/TNF- $\alpha$-induced genes and the psoriasis gene signature. We previously reported the more synergistic activities of the combined stimulation of TNF- $\alpha$, IL-17A, and IFN- $\gamma$ in epidermal keratinocytes [32]. For example, the triple stimulation synergistically induces IL-17C, IL-36 $\gamma$, and the antimicrobial peptide human b-defensin-2 in epidermal keratinocytes [32]. Therefore, we predicted that normal human epidermal keratinocytes (NHEKs) stimulated with the triple cytokines would mimic the condition of psoriatic keratinocytes. Epidermal keratinocytes were already reported to express IL23Ap19 and EBI3 mRNAs after stimulation with poly (I:C) [22].
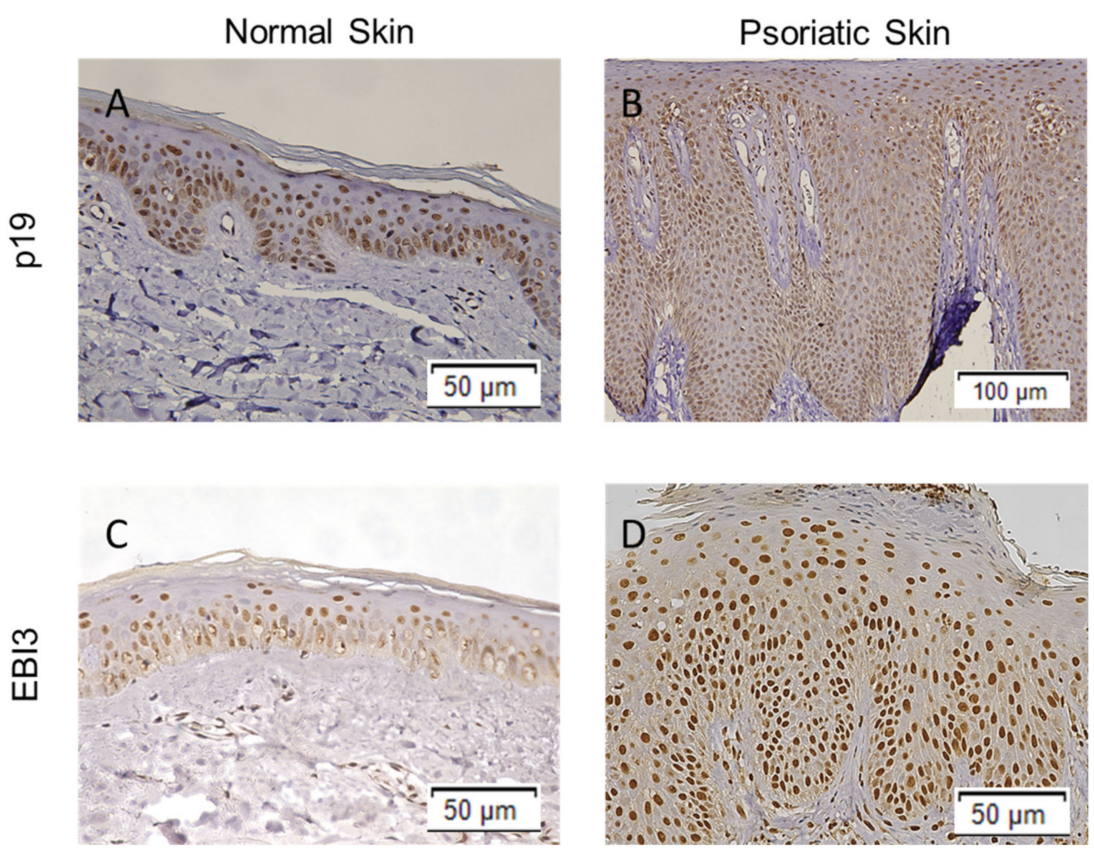

Figure 1. Expressions of IL-23Ap19 and EBI3 are upregulated in psoriatic keratinocytes. Expressions of IL-23Ap19 and EBI3 were examined in normal skin and lesional psoriatic skin sections by immunohistochemistry with polyclonal rabbit anti-IL-23p19 antibody $(\mathbf{A}, \mathbf{B})$ and monoclonal mouse anti-EBI3 antibody $(\mathbf{C}, \mathbf{D})$.

First, we stimulated NHEKs with TNF- $\alpha$, IL-17A, or IFN- $\gamma$ alone to investigate whether NHEKs express the genes IL23Ap19 and EBI3 under conditions other than poly (I:C) stimulation. TNF- $\alpha$ and IL-17A but not IFN- $\gamma$ significantly induced expression of the IL23Ap19 mRNA, and TNF- $\alpha$ and IFN- $\gamma$, but not IL-17A induced expression of the EBI3 mRNAs in the cells (Figure 2A,B). As expected, triple stimulation of the cytokines (TNF- $\alpha$, IL-17A, and IFN- $\gamma$ ) maximally induced the expression of both IL23Ap19 and EBI3 (Figure 2C,D). Other triple-stimulation combinations, such as TNF- $\alpha /$ IL-17A/IFN- $\alpha$, TNF$\alpha /$ IL-17A/IL-27, and TNF- $\alpha /$ IL-17A/IL-17C, did not express IL23Ap19 and EBI3 mRNAs as strongly as that of TNF- $\alpha$, IL-17A, and IFN- $\gamma$ (Figure 2C,D). IL12Ap35 and IL27Ap28 were undetectable in NHEKs by qPCR in our culture systems (data not shown).

\subsection{Topical Treatment by Dexamethasone, Vitamin D3, and Acitretin Suppress p19 and EBI3 Expression in Epidermal Keratinocytes}

Topical steroids, vitamin D3 ointment, and topical and oral synthetic retinoid are clinically used as standard treatments for mild-to-moderate psoriasis. We next investigated whether the expressions of IL23Ap19 and EBI3 are suppressed with dexamethasone, vitamin D3, and acitretin in NHEKs. Dexamethasone significantly suppressed the expression of 
IL23Ap19 mRNAs, but not EBI3 mRNAs in the cells (Figure 3A,B). Vitamin D3 and acitretin significantly suppressed the expression of both IL23Ap19 and EBI3 mRNAs in a dosedependent manner (Figure 3C-F).

A

C

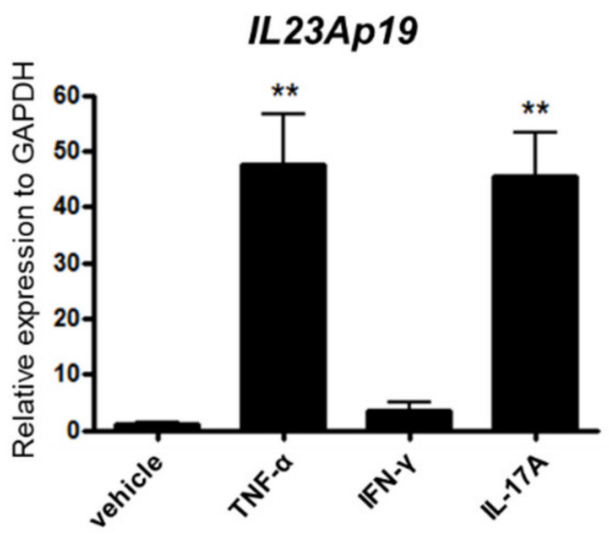

IL23Ap19

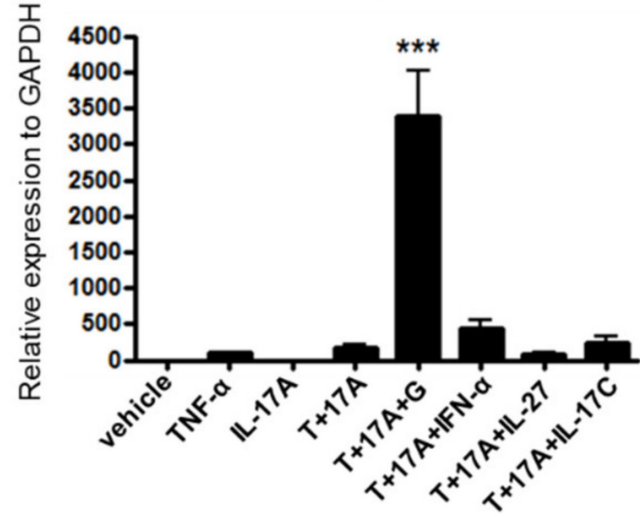

B

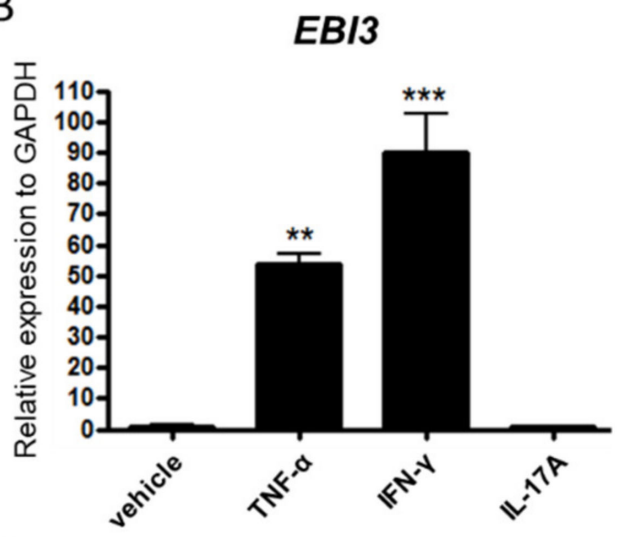

D

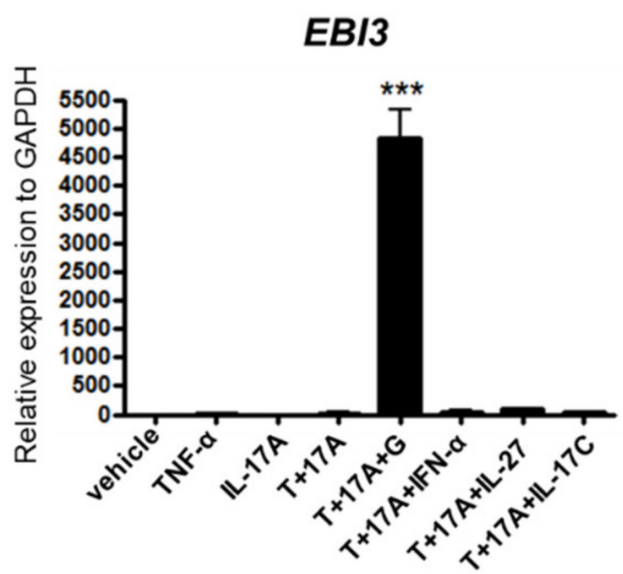

Figure 2. TNF- $\alpha$, IL-17A, and IFN- $\gamma$ synergistically induce IL23Ap19 and EBI3 expressions in NHEKs. (A-D) Normal human epidermal keratinocytes (NHEKs) were stimulated with (A,B) TNF- $\alpha$ (50 ng/mL), IL-17A (50 ng/mL), or IFN- $\gamma(50 \mathrm{ng} / \mathrm{mL})$, and (C,D) triple cytokine combinations of TNF- $\alpha$, IL-17A, IFN- $\gamma$, IFN- $\alpha$, IL-27 and/or IL-17C for $24 \mathrm{~h}$. The relative mRNA expressions of IL23Ap19 and EBI3 to GAPDH were analyzed with quantitative real-time PCR (qPCR). ${ }^{* *} p<0.01$, *** $p<0.001, \mathrm{~T}, \mathrm{TNF}-\alpha ; 17 \mathrm{~A}, \mathrm{IL}-17 \mathrm{~A} ; \mathrm{G}, \mathrm{IFN}-\gamma$.

\subsection{The Analysis of IL-23Ap19 and EBI3 Protein Expressions in Epidermal Keratinocytes}

We further used ELISA to examine whether NHEKs produce IL-23Ap19- and/or EBI3-including cytokines, such as IL-23 (p19/p40), IL-27 (p28/EBI3), IL-35 (p35/EBI3), and IL-39 (p19/EBI3). The expressions of IL-23Ap19 and EBI3 proteins were significantly enhanced under the triple stimulation of TNF- $\alpha$, IL-17A, IFN- $\gamma$ in NHEKs (Figure 4A,B). However, we were not able to detect IL-23 or IL-39 by ELISA in the stimulated cells (data not shown). IL-27 and IL-35 were also undetected (data not shown).

To further investigate what the IL-23Ap19 and EBI3 produced by NHEKs bind to, we immunoprecipitated the supernatant of keratinocytes stimulated with the triple combination (TNF- $\alpha$, IL-17A, and IFN- $\gamma$ ), using tildrakizumab (humanized anti-human IL-23Ap19 antibody) and rabbit anti-human EBI3 antibody, and analyzed the proteins by LC-Ms/Ms. IL-23Ap19 was appropriately detected in the protein complexes immunoprecipitated with tildrakizumab, but EBI3 and p40 were not detected (Figure 5A or Figures S1 and S2). Although the analysis system also detected miscellaneous proteins, none of the proteins were prone to be paired with IL-23Ap19. We detected EBI3 in the protein complexes 
immunoprecipitated with anti-human EBI3 antibody, but the IL-23Ap19 protein was not included (Figures 5B, S3 and S4).

A

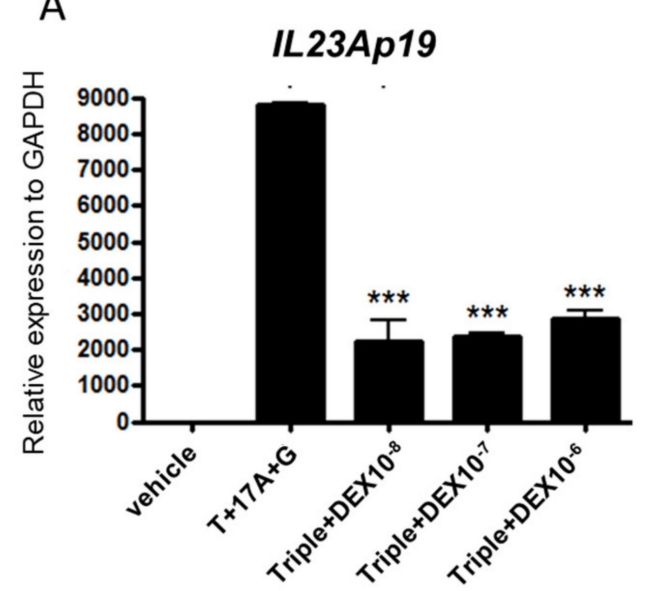

C

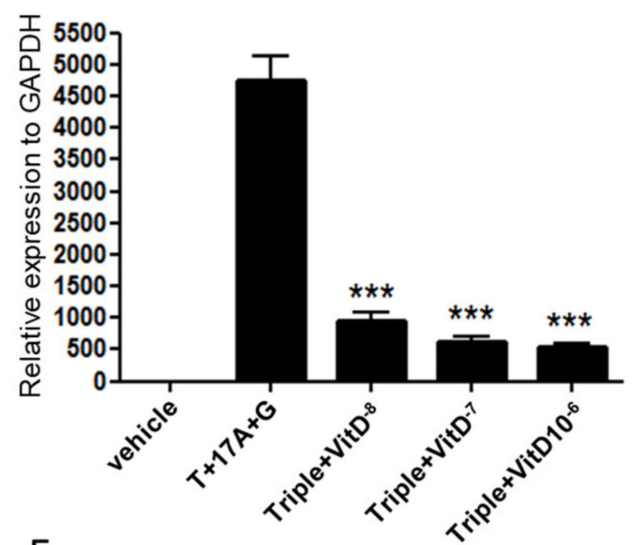

E

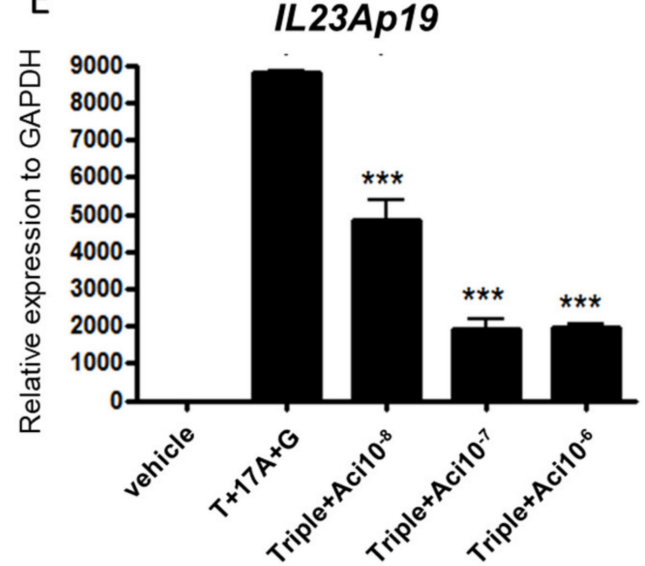

B

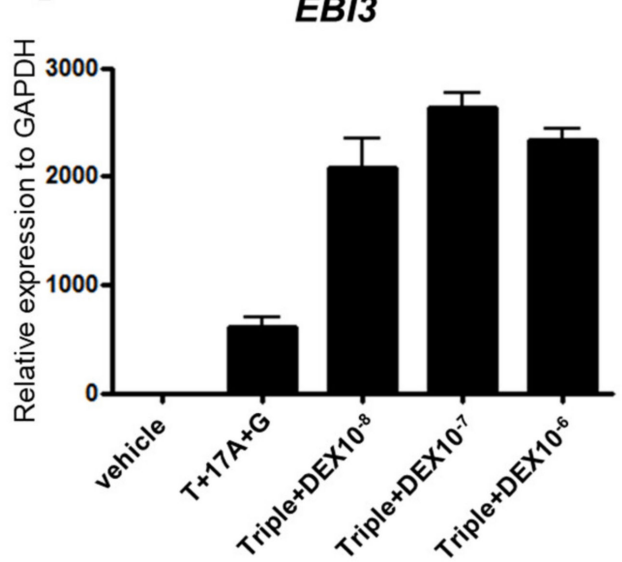

D

EBI3
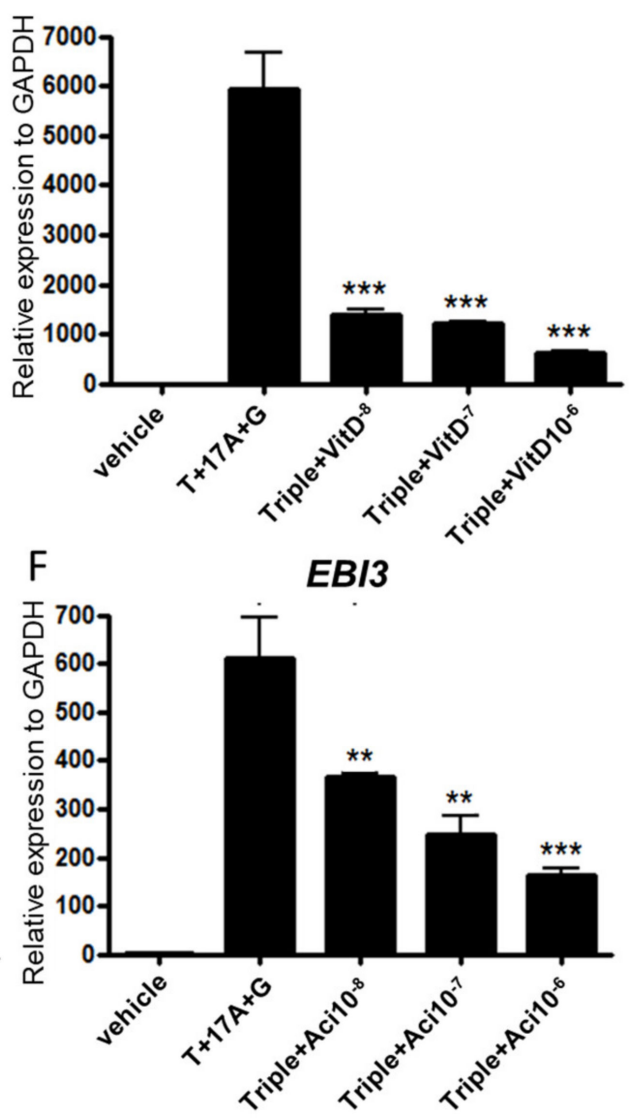

Figure 3. Topical treatment by dexamethasone, vitamin D3, and acitretin suppress IL23Ap19 and EBI3 expressions in NHEKs. Normal human epidermal keratinocytes (NHEKs) were stimulated with the triple combination of TNF- $\alpha(50 \mathrm{ng} / \mathrm{mL})$, IL-17A $(50 \mathrm{ng} / \mathrm{mL})$, and IFN- $\gamma(50 \mathrm{ng} / \mathrm{mL})$, and simultaneously treated with (A,B) dexamethasone (10-8,10-7, or 10-6 M), (C,D) 1,25(OH)2 vitamin D3 (10-8,10-7, or 10-6 M), or (E,F) acitretin (10-8,10-7, or 10-6 M) for $24 \mathrm{~h}$. The relative mRNA expressions of IL23Ap19 and EBI3 to GAPDH were analyzed with quantitative real-time PCR (qPCR). ${ }^{* *} p<0.01$, *** $p<0.001$. T, TNF- $\alpha$; 17A, IL-17A; G, IFN- $\gamma$; Triple, T+17A+G; DEX, dexamethasone; VitD, 1,25(OH)2 vitamin D3; Aci, acitretin. 
A

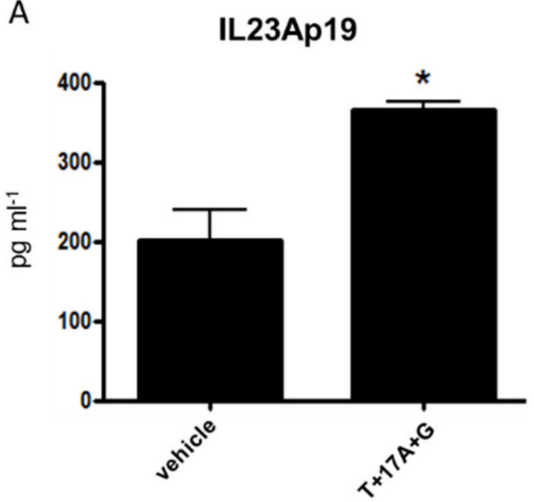

B

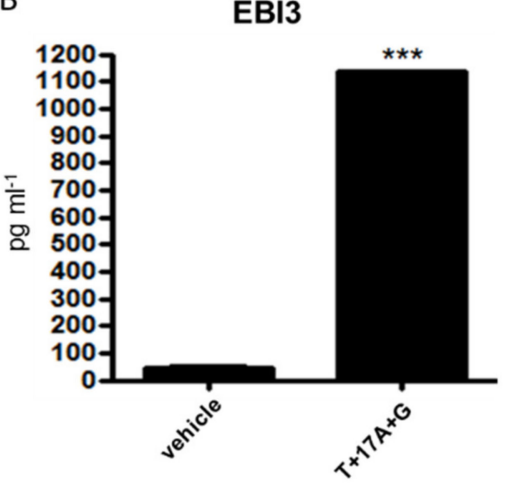

Figure 4. Analysis of IL-23Ap19 and EBI3 protein expressions in epidermal keratinocytes. Normal human epidermal keratinocytes (NHEKs) were stimulated with the triple combination of TNF- $\alpha$ $(50 \mathrm{ng} / \mathrm{mL})$, IL-17A (50 ng/mL) and IFN- $\gamma(50 \mathrm{ng} / \mathrm{mL})$ for $24 \mathrm{~h}$. ELISA was used to measure protein levels of (A) IL-23Ap19 and (B) EBI3. ${ }^{*} p<0.05,{ }^{* * *} p<0.001$. T, TNF- $\alpha$; 17, IL-17A; G, IFN- $\gamma$.

A

\begin{tabular}{|c|c|c|c|c|c|c|c|c|c|c|}
\hline Group & Spectra & Distinct & Distinct & $\% \mathrm{AA}$ & Mean & Protein MW & Protein & Species & Database & Protein Name \\
\hline & & Peptides & Summed & Coverage & Peptide & (Da) & $\mathrm{pl}$ & & Accession & \\
\hline & & & MS/MS Search & & Spectral & & & & & \\
\hline & & & Score & & Intensity & & & & & \\
\hline 1 & 3 & 1 & 19.40 & 3.1 & 4980000.00 & 47686.4 & 5.1 & HUMAN & Q13501 & Sequestosome-1 \\
\hline 2 & 2 & 1 & 15.90 & 5.2 & 252000000.00 & 20729.4 & 6.02 & HUMAN & Q9NPF7 & Interleukin-23 subunit alpha \\
\hline 3 & 1 & 1 & 14.80 & 11.4 & 83200000.00 & 13241.8 & 5.71 & HUMAN & P06702 & Protein S100-A9 \\
\hline 4 & 1 & 1 & 13.30 & 1.7 & 4070000.00 & 70051 & 5.48 & HUMAN & P0DMV8 & Heat shock $70 \mathrm{kDa}$ protein $1 \mathrm{~A}$ \\
\hline 5 & 1 & 1 & 12.89 & 6 & 954000.00 & 23431.4 & 10.94 & HUMAN & P50914 & 60S ribosomal protein L14 \\
\hline 6 & 1 & 1 & 12.39 & 0.4 & 13500000.00 & 240849.4 & 4.79 & HUMAN & P24821 & Tenascin \\
\hline 7 & 1 & 1 & 12.20 & 8.1 & 33800000.00 & 10880.9 & 9.93 & HUMAN & P02778 & C-X-C motif chemokine 10 \\
\hline Totals: & 10 & th & & & & & & & & \\
\hline
\end{tabular}

B

\begin{tabular}{|c|c|c|c|c|c|c|c|c|c|c|}
\hline Group & Spectra & Distinct & Distinct & $\%$ AA & Mean & \begin{tabular}{|l|} 
Protein MW \\
\end{tabular} & Protein & Species & Database & Protein Name \\
\hline & & Peptides & Summed & Coverage & Peptide & $(\mathrm{Da})$ & $\mathrm{pl}$ & & Accession & \\
\hline & & & MS/MS Search & & Spectral & & & & & \\
\hline & & & Score & & Intensity & & & & & \\
\hline 1 & 5 & 5 & 66.29 & 14.8 & 62800000.00 & 51560.6 & 5.09 & HUMAN & P02533 & Keratin, type I cytoskeletal 14 \\
\hline 2 & 4 & 2 & 37.11 & 16.8 & 67700000.00 & 19778.8 & 4.71 & HUMAN & 014950 & Myosin regulatory light chain $12 \mathrm{~B}$ \\
\hline 3 & 3 & 3 & 36.02 & 10.6 & 74300000.00 & 22579.8 & 10.91 & HUMAN & P16401 & Histone $\mathrm{H} 1.5$ \\
\hline 4 & 2 & 2 & 30.24 & 15.7 & 179000000.00 & 27744.1 & 10.37 & HUMAN & Q07955 & Serine/arginine-rich splicing factor 1 \\
\hline 5 & 1 & 1 & 20.68 & 5.3 & 36900000.00 & 39616.3 & 9.8 & HUMAN & 075367 & Core histone macro-H2A.1 \\
\hline 6 & 1 & 1 & 20.52 & 6.7 & 79500000.00 & 20862.5 & 10.84 & HUMAN & P07305 & Histone H1.0 \\
\hline 7 & 1 & 1 & 19.14 & 28.6 & 129000000.00 & 11664.7 & 4.42 & HUMAN & P05387 & 60 S acidic ribosomal protein $\mathrm{P} 2$ \\
\hline 8 & 1 & 1 & 18.16 & 5.2 & 256000000.00 & 25395.9 & 9.41 & HUMAN & Q14213 & Interleukin-27 subunit beta \\
\hline 9 & 1 & 1 & 17.71 & 16.8 & 34900000.00 & 13281.3 & 11.57 & HUMAN & P62314 & Small nuclear ribonucleoprotein Sm D1 \\
\hline 10 & 1 & 1 & 17.56 & 1.5 & 66800000.00 & 95336.7 & 8.85 & HUMAN & Q12906 & Interleukin enhancer-binding factor 3 \\
\hline 11 & 1 & 1 & 16.68 & 20.1 & 15000000.00 & 11831.8 & 10.63 & HUMAN & P52926 & High mobility group protein $\mathrm{HMGI}-\mathrm{C}$ \\
\hline 12 & 2 & 1 & 16.52 & 4.7 & 360000000.00 & 32574.5 & 4.64 & HUMAN & P06748 & Nucleophosmin \\
\hline 13 & 1 & 1 & 15.09 & 8.2 & 83500000.00 & 22782.1 & 5.98 & HUMAN & P04792 & Heat shock protein beta-1 \\
\hline 14 & 1 & 1 & 14.82 & 8 & 142000000.00 & 27118 & 8.43 & HUMAN & Q9BRN9 & TM2 domain-containing protein 3 \\
\hline 15 & 1 & 1 & 14.64 & 6.2 & 149000000.00 & 22763.6 & 5.56 & HUMAN & P14649 & Myosin light chain 6B \\
\hline 16 & 1 & 1 & 14.55 & 11.7 & 117000000.00 & \begin{tabular}{|c|}
15074.8 \\
\end{tabular} & 7.02 & HUMAN & P47929 & Galectin-7 \\
\hline 17 & 1 & 1 & 13.98 & 12 & 73900000.00 & $\begin{array}{c}9989.5 \\
\end{array}$ & 9.27 & HUMAN & P13501 & C-C motif chemokine 5 \\
\hline 18 & 2 & 1 & 13.72 & 0.7 & 114000000.00 & 163288.1 & 6.03 & $\begin{array}{l}3 \text { HUMAN } \\
\end{array}$ & P01023 & Alpha-2-macroglobulin \\
\hline 19 & 1 & 1 & 12.78 & 10.4 & 104000000.00 & 12783.8 & 9.65 & HUMAN & P62888 & 60S ribosomal protein $\mathrm{L} 30$ \\
\hline 20 & 1 & 1 & 12.2 & 11.2 & 172000000.00 & 15422.9 & 10.79 & $\begin{array}{l}\text { HUMAN } \\
\text { HUM }\end{array}$ & P62847 & 40 S ribosomal protein S24 \\
\hline 21 & 2 & 1 & 12.02 & 10.7 & 66500000.00 & 14865.2 & 10.5 & HUMAN & P62829 & 60S ribosomal protein $\mathrm{L} 23$ \\
\hline 22 & 1 & 1 & 11.56 & 2.7 & 49700000.00 & 49670 & 4.78 & HUMAN & P07437 & Tubulin beta chain \\
\hline 23 & 1 & 1 & 11.08 & 11.1 & 44400000.00 & 15859.5 & 11.32 & HUMAN & P62910 & 60S ribosomal protein $\mathrm{L} 32$ \\
\hline Totals: & 36 & 31 & & & & & & & & \\
\hline
\end{tabular}

Figure 5. Proteins detected by immunoprecipitation with tildrakizumab or anti-human EBI3 antibody and LC-Ms/Ms. Culture supernatant of NHEKs stimulated with the triplet mixture of cytokines (50 ng/mL, TNF- $\alpha$, IL-17A and IFN- $\gamma$ ) for $48 \mathrm{~h}$ were immunoprecipitated with (A) humanized anti-human IL-23Ap19 antibody or (B) rabbit anti-human EBI3 antibody and magnetic beads. The detected proteins were analyzed by liquid chromatography-electrospray tandem mass spectrometry (LC-Ms/Ms). The non-specific proteins detected in common with the control were removed. 
Collectively, our results suggest that human epidermal keratinocytes produced IL23Ap19 and EBI3 proteins but not the heterodimeric cytokines IL-23, IL-27, IL-35, or IL-39.

\section{Discussion}

IL-23 and IL-39 have been reported as p19-including heterodimeric cytokines. On the other hand, IL-27, IL-35, and IL-39 are known to be EBI3-including cytokines. In this study, we focused on p19- and/or EBI3-including cytokines produced by psoriatic keratinocytes. Our immunohistochemical analysis showed that the expressions of p19 and EBI3 are increased in psoriatic keratinocytes. We also confirmed that cultured epidermal keratinocytes had elevated IL-23Ap19 and EBI3 mRNA and protein when stimulated with the triple combination of TNF- $\alpha$, IL-17A, and IFN- $\gamma$, which mimics psoriatic inflammation. Moreover, the expressions of IL-23Ap19 and EBI3 were suppressed by dexamethasone, vitamin D3, and acitretin, which are commonly used for psoriasis therapy. However, our ELISA experiment and LC-Ms/Ms analyses did not detect the heterodimeric cytokines IL-23 (p19/p40), IL-27 (p28/EBI3), IL-35 (p35/EBI3), or IL-39 (p19/EBI3) in epidermal keratinocytes with triple stimulation. These findings suggest that the IL-23Ap19 and EBI3 produced by epidermal keratinocytes may not form heterodimeric cytokines IL-23, IL-27, IL-35, or IL-39 and may exist as a monomer or homopolymer, such as a homodimer or homotrimer, as shown in Figure 6.

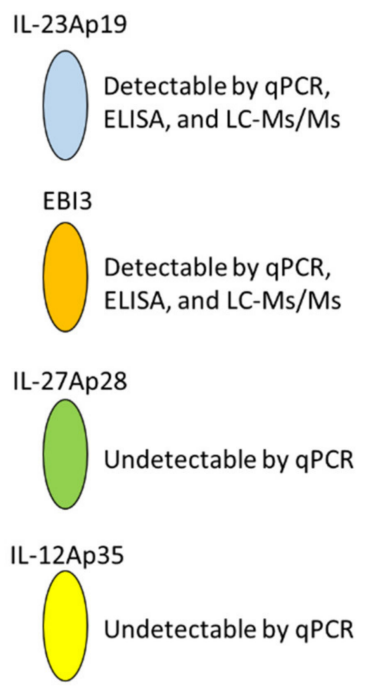

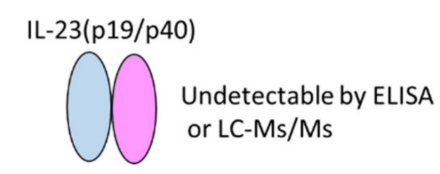
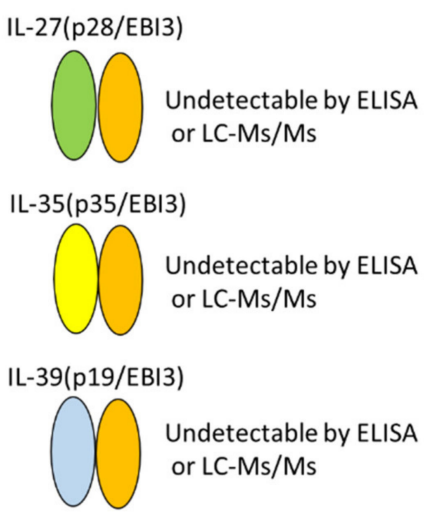

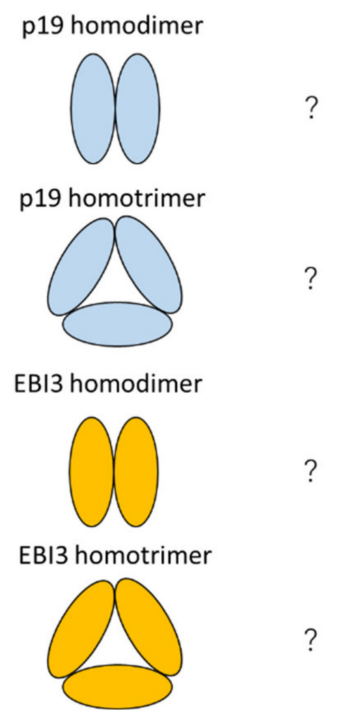

Figure 6. IL-23Ap19- and/or EBI3-including cytokines in epidermal keratinocytes. IL-23Ap19 and EBI3 were detected by qPCR, ELISA, and LC-Ms/Ms. IL-27Ap28 and IL-12Ap35 were undetected by qPCR. IL-23(p19/p40), IL-27(p28/EBI3), IL-35(p35/EBI3), and IL-39(p19/EBI3) were undetected by ELISA or LC-Ms/Ms. This result raises the possibility that IL-23Ap19 and EBI3 produced from epidermal keratinocytes exist as a monomer or homopolymer, such as a homodimer or homotrimer. ?, Not yet detected and possibility.

The IL-12 family is unique in having heterodimeric cytokines, and this promiscuous pairing of $\alpha / \beta$ subunits is speculated to contribute to the dual role of inflammatory and anti-inflammatory cytokines. One of the roles of the two subunits of the IL-12 cytokine family is to promote the extracellular secretion of molecules that are difficult to secrete extracellularly [28]. In other words, IL-23Ap19 and EBI3 are not easily secreted as a monomer or homopolymer, but when they form a heterodimer, they are secreted extracellularly and can function as a cytokine. However, there seems to be a difference in the ease of secretion depending on the type of IL-12 cytokine family. In a study revisiting the combinatorial potential of cytokine subunits in the IL-12 family using HEK293 T cells co-expressing a set of each possible $\alpha / \beta$ subunit combination, IL-12 and IL-23 were extensively secreted, but IL-27 and IL-35 were poorly secreted [28]. The $\alpha$-subunits p19, p28, and p35 were 
retained inside the cell; on the other hand, the $\beta$-subunits p40 and EBI 3 could be secreted independently. Meanwhile, p40, a $\beta$-subunit of IL-12 or IL-23, is able to form a homodimer (p80) [33]. The p40 homodimer (p80) binds to an IL-12 receptor, but its role remains poorly understood, due to the lack of specific monoclonal antibodies. p28, an $\alpha$-subunit of IL-27, is also called IL-30 and remains functional, even in the absence of EBI3. In particular, the p28 monomer was shown to suppress anti-allogeneic immune responses and to antagonize cytokine signaling through the gp130- and IL-6-mediated production of IL-17 and IL-10 [34,35]. From these facts, IL-23Ap19 and EBI3 may form a homodimer or remain functional even as monomers, although no report prior to this has suggested that possibility.

The expression of $E B I 3$ and $p 19$ was also demonstrated by human intestinal epithelial cells [36]. However, the cells did not co-express $p 40$ or $p 28$ that are required to form IL-23 or IL-27, respectively. Taken together with our results, the expression forms of IL23Ap19- and/or EBI3-including cytokines in epithelial cells may be different from those in immune cells.

EBI3, discovered in B lymphocytes infected with Epstein-Barr virus in 1996, regulates cell-mediated immune responses [37]. EBI3 expression has also been described in placental syncytiotrophoblasts, endothelial cells, plasma cells, etc. EBI3 downregulation contributes to type I collagen overexpression in scleroderma skin, although there have been no reports about the association between psoriasis and EBI3 [38]. Thus, we are the first to show the association and up-regulation of EBI3 in psoriatic skin, its production by NHEKs stimulated with TNF- $\alpha$, IL-17A, IFN- $\gamma$, and its suppression by dexamethasone, vitamin D3, and acitretin. Further investigation is needed to clarify the detailed roles of EBI3 in psoriatic pathology and the novel properties of EBI3. EBI3 might be a therapeutic target for psoriasis in the future.

IL-23, an essential cytokine for the pathology and the treatment of psoriasis, is mainly produced by macrophages and dendritic cells, and has a crucial role for immunity to mycobacterial and fungal infection $[14,39,40]$. Macrophages and dendritic cells are the primary cell types responsible for the overexpression of IL-23 in psoriatic pathogenesis [39]. On the other hand, some research groups have reported the production of IL-23 by human keratinocytes, in contrast to our results. Ehst et al. reported that IL-23 protein was enhanced upon stimulation of human keratinocytes by TNF- $\alpha$ plus IL-17A [41]. Li et al. showed that keratinocytes could produce IL-23 at levels sufficient to cause differentiation of IL-17Aproducing T cells and skin inflammation in a mouse model [13]. Although we successfully confirmed that epidermal keratinocytes had increased expressions of IL-23Ap19 [9-13], we were not able to detect IL-23 in the cells with either ELISA or LC-Ms/Ms. Epidermal keratinocytes might express IL-23Ap19 as a monomer or homopolymer such as homodimer or homotrimer rather than IL-23.

The role and function of IL-39 are still not entirely clear. There are still very few reports about IL-39, and some reports even deny its existence in humans. However, it seems that HEK293 cells transfected with IL-23Ap19 and EBI3 secrete IL-23Ap19/EBI3 heterodimers [22,27]. Similar to our findings, Ecoeur et al. reported the elevated expression of IL-23Ap19 and EBI3 mRNAs in human keratinocytes, although they failed to detect the formation of IL-23Ap19/EBI3 heterodimers in response to stimulation of neutrophils and peripheral blood mononuclear cells [27]. They concluded that the secretion of IL23Ap19/EBI3 complexes could be forced in human cells, although the complexes may be secreted below the lower limit of detection or have no functional role. We agree with their conclusions and believe that IL-39 is unlikely to be produced by epidermal keratinocytes and unlikely to be involved in psoriatic pathogenesis.

Anti-IL-23Ap19 antibodies are characterized by their long-lasting and highly effective nature and are not inferior to anti-human IL-17A antibodies [42]. Tildrakizumab (humanized anti-human IL-23Ap19 antibody) can correctly identify the keratinocyte-derived IL-23Ap19 protein. Anti-human IL-23Ap19 antibodies may also be more effective rather than anti-IL-12/23p40 antibodies by binding to keratinocyte-derived IL-23Ap19 protein. 
Elucidation of the role of the IL-23Ap19 monomer or homopolymer, such as that of a homodimer or homotrimer, might contribute more profoundly to our understanding of the pathology and potential treatments of psoriasis.

In conclusion, we demonstrated that epidermal keratinocytes produce IL-23Ap19 and EBI3 proteins but did not detect the heterodimeric cytokines IL-23, IL-27, IL-35, or IL-39 in the cells. IL-23Ap19 and EBI3 produced from epidermal keratinocytes may exist as a monomer or homopolymer, such as a homodimer or homotrimer. Further investigation of the role of keratinocyte-derived IL-23Ap19 and EBI3 is required to clarify the pathogenesis of psoriasis.

\section{Subjects and Methods}

\subsection{Immunohistochemistry}

The present study was approved by the Institutional Review Board (IRB) of Okayama University Hospital (No. 1712-018). Extracutaneous biopsy specimens obtained from psoriasis patients for diagnostic use were subjected to immunostaining. Normal skin samples were obtained from healthy volunteers at Okayama University Hospital. Written informed consent was obtained from all tissue donors according to the Helsinki Declaration. Formalin-fixed, paraffin-embedded skin samples were cut into $4 \mu \mathrm{m}$ sections and mounted on glass slides. The slides were deparaffinized and activated with citric acid buffer ( $0.01 \mathrm{~mol} / \mathrm{L}, \mathrm{pH}$ 6.0; LSI Medience Corporation, Tokyo, Japan) for $5 \mathrm{~min}$ at $90{ }^{\circ} \mathrm{C}$ in a pressure cooker. They were then incubated with peroxidase-blocking solutions (Dako, Glostrup, Copenhagen, Denmark) for $10 \mathrm{~min}$ at room temperature and blocking solution (Life Technologies, Carlsbad, CA, USA) at $4{ }^{\circ} \mathrm{C}$ overnight. Next, the sections were incubated with rabbit polyclonal anti-human IL-23Ap19 antibody (MBS240347; MyBio Source, San Diego, CA, USA) or mouse monoclonal anti-human IL-27/IL-35 EBI3 antibody (MAB6456; R\&D Systems, Minneapolis, MN, USA) at $4{ }^{\circ} \mathrm{C}$ overnight. Finally, the slides were incubated with goat anti-mouse/rabbit IgG antibodies conjugated to peroxidaselabeled polymer (Dako, Tokyo, Japan) for $30 \mathrm{~min}$. Histochemical visualization was carried out with 3,3-diaminobenzidine (DAB) (Dako).

\subsection{Cell Culture and Stimuli}

Normal human epidermal keratinocytes (NHEKs) were obtained from Cascade Biologics/Invitrogen (catalog number C-001-5C; Portland, OR, USA) and grown in serum-free EpiLife cell culture media (Cascade Biologics/Invitrogen) containing $0.06 \mathrm{mM} \mathrm{Ca}^{2+}$ and $1 \times$ EpiLife Defined Growth Supplement (EDGS; Cascade Biologics/Invitrogen, Carlsbad, CA, USA) at $37^{\circ} \mathrm{C}$ under standard tissue culture conditions. Cultures were maintained for up to nine passages in this media with the addition of $100 \mathrm{U} / \mathrm{mL}$ penicillin, $100 \mu \mathrm{g} / \mathrm{mL}$ streptomycin, and $0.25 \mu \mathrm{g} / \mathrm{mL}$ amphotericin B. NHEKs were grown in 24-well flat-bottom plates (Corning Incorporated, Corning, NY, USA). Upon reaching 70-100\% confluence, cells were stimulated and/or treated with one or more of the following: IL-4 $(50 \mathrm{ng} / \mathrm{mL} ; \mathrm{R} \& \mathrm{D}$ Systems, Minneapolis, MN, USA), IL-17A ( $50 \mathrm{ng} / \mathrm{mL}$; R \& D systems), IL-17C (50 ng/mL; R\&D systems), IL-22 (50 ng/mL; R\&D Systems), IL-27 (50 ng/mL; R\&D Systems), IL-29 (50 ng/mL; Proteintech, Rosemont, IL, USA), TNF- $\alpha$ ( $50 \mathrm{ng} / \mathrm{mL}$; eBioscience, San Diego, CA, USA), IFN- $\gamma$ (50 ng/mL; R\&D Systems), dexamethasone (10-8,10-7, or 10-6 M; SigmaAldrich, MO, USA), 1,25(OH)2 vitamin D3 (10-8,10-7, or 10-6 M; Sigma-Aldrich), and synthetic retinoid acitretin (10-8,10-7, or 10-6 M; Sigma-Aldrich), using phosphate-buffered saline (PBS) as a vehicle for up to $24 \mathrm{~h}$. After cell stimulation/treatment, culture supernatants were collected and stored at $-20^{\circ} \mathrm{C}$ until analysis. RNA from NHEKs was extracted using TRIzol reagent (Invitrogen). RNA was stored at $-80^{\circ} \mathrm{C}$ until analysis.

\subsection{Quantitative Real-Time PCR}

Complementary DNA (cDNA) was synthesized from RNA using an iScript cDNA Synthesis Kit (BioRad, Hercules, CA, USA) according to the manufacturer's protocol. TaqMan gene Expression Assays (Applied Biosystems ABI, Foster City, CA, USA) were used 
to analyze the expression of human EBI3 (assay ID: Hs01057148_m1), IL12A (assay ID: Hs01073447), IL23Ap19 (assay ID: Hs00372324_m1), and IL27A (assay ID: Hs00377366) according to the manufacturer's protocol (User Bulletin \#2, Applied Biosystems). Glyceraldehyde 3-phosphate dehydrogenase (GAPDH) was used as an internal control to validate the RNA for each cultured keratinocyte sample. GAPDH mRNA was detected using the VIC-CAT CCA TGA CAA CTT TGG TA-MGB probe with the primers $5^{\prime}$-CTT AGC ACC CCT GGC CAA G-3' and 5'-TGG TCA TGA GTC CTT CCA CG-3'. Each mRNA expression was calculated as the expression relative to GAPDH mRNA, and all data are presented as fold changes in comparison with the control (the mean value of the non-stimulated cells).

\subsection{Enzyme-Linked Immunosorbent Assay (ELISA)}

Protein levels of EBI3 (Aviva Systems Biology, San Diego, CA, USA), IL-23Ap19 (Aviva Systems Biology), IL-23 (eBioscience, San Diego, CA, USA), IL-27 (R\&D systems), IL-35 (AVIVA system biology), and IL-39 (MyBioSource, San Diego, CA, USA) in culture supernatants were measured by a commercial sandwich ELISA, according to the manufacturer's protocol. Absorbance at $450 \mathrm{~nm}$ was determined using a microplate reader (SH-1000Lab; Corona Electric, Hitachinaka, Ibaraki, Japan).

\subsection{Liquid Chromatography-Electrospray Tandem Mass Spectrometry (LC-Ms/Ms)}

The collected condition media from NHEKs that were stimulated with the triplet mixture of cytokines (50 ng/mL, TNF- $\alpha$, IL-17A and IFN- $\gamma$ ) for $48 \mathrm{~h}$ were treated with rabbit anti-human EBI3 antibody (119-16298; Raybiotech, Peachtree Corners, GA, USA) or humanized anti-human IL-23p19 antibody (tildrakizumab, kindly provided by Sun Pharmaceutical Industries) or neither for $16 \mathrm{~h}$ at $4{ }^{\circ} \mathrm{C}$. Protein G-coated magnetic beads (Sure BeadsTM Protein G Magnetic Beads; BioRad Laboratories, Hercules, CA, USA) were then added into the individual specimens and gently mixed by a rotating procedure for another $2 \mathrm{~h}$ at the same temperature. Magnetic immunoprecipitation (mag-IP) that allowed the beads to absorb the protein complexes (antibody-antigen and antigen bound proteins) was performed for the treated specimens according to the conventional procedure. The collected immunoprecipitates were eluted from the beads by an acidic buffer (100 mM of Glycin- $\mathrm{HCl}$ buffer/pH 2.0), neutralized with $1.0 \mathrm{M}$ of Tris- $\mathrm{HCl}$ buffer/pH 9.0, dialyzed with trypsin digestion buffer $\left(10 \mathrm{mM} \mathrm{CaCl}_{2}, 100 \mathrm{mM}\right.$ of ammonium bicarbonate/pH 7.8), and trypsinized overnight at $37{ }^{\circ} \mathrm{C}$. The digested proteins were directly subjected to a shotgun-type protein identification using a nano-flow liquid chromatography-mass spectrometry apparatus (Agilent 6330 Ion Trap; Agilent Technologies, Santa Clara, CA, USA) equipped with an analytical chip (Agilent HPLC-Chip; Agilent Technologies). The resulting tandem mass spectrometry spectra of the tryptic peptides were finally analyzed using Agilent software (Spectrum Mill MS Proteomics Workbench; Agilent Technologies) with the protein database (SwissPlot) for putative Homo sapiens protein identifications. The non-specific proteins detected in common with the control were removed.

\subsection{Statistical Analysis}

Results were expressed as means \pm SEMs. Student's t-test was used to determine the significance of differences between groups. One-way analysis of variance with Tukey's test was used to determine significance among more than two groups. The analyses were performed by GraphPad Prism 4 (GraphPad Software, San Diego, CA, USA). $p$-values $<0.05$ were considered to indicate significance.

\section{Conclusions}

Epidermal keratinocytes produced both IL-23Ap19 and EBI3 proteins, but we did not detect the heterodimeric cytokines IL-23, IL-27, IL-35, or IL-39. Tildrakizumab correctly identified the keratinocyte-derived IL-23Ap19 protein. IL-23Ap19 and EBI3 produced from keratinocytes may exist as a monomer or homopolymer, such as a homodimer or 
homotrimer. Further investigation is required to clarify the function of keratinocyte-derived IL-23Ap19 and EBI3 in psoriasis.

Supplementary Materials: The following are available online at https:/ /www.mdpi.com/article/ $10.3390 / \mathrm{ijms} 222312659 / \mathrm{s} 1$, all proteins detected by immunoprecipitation with tildrakizumab or anti-human EBI3 antibody and LC-Ms/Ms.

Author Contributions: K.T. and S.M. designed this study. K.T., N.T. (Nina Tang), H.U., A.K., F.J., N.T. (Nahoko Tomonobu) and M.S. performed the experiments. M.K., A.K., H.N., M.S. (Minori Sasakura) and S.S. contributed to sample preparation. K.T., M.S. (Masakiyo Sakaguchi), M.O. and S.M. contributed to the interpretation of the results. K.T. and S.M. wrote the paper with input from all authors. All authors have read and agreed to the published version of the manuscript.

Funding: This work was supported by a Grant-in-Aid for Scientific Research (C) (no. 20K08672) and a Grant-in-Aid for Research Activity Start-up (No. 20K22739).

Institutional Review Board Statement: The present study was approved by the Institutional Review Board (IRB) of Okayama University Hospital (No. 1712-018).

Informed Consent Statement: Informed consent was obtained from all subjects involved in the study.

Data Availability Statement: The data that support the findings of this study are available from the corresponding author, upon reasonable request.

Conflicts of Interest: The authors declare no conflict of interest.

\begin{tabular}{ll}
\multicolumn{2}{l}{ Abbreviations } \\
EBI3 & Epstein-Barr virus-induced 3 \\
ELISA & Enzyme-linked immunosorbent assay \\
GAPDH & Glyceraldehyde 3-phosphate dehydrogenase \\
IFN & Interferon \\
IL & Interleukin \\
LC-Ms/Ms & Liquid chromatography-electrospray tandem mass spectrometry \\
NHEKs & Normal human epidermal keratinocytes \\
Poly (I:C) & Polycytidylic acid \\
RT-PCR & Real-time polymerase chain reaction \\
STAT & Signal transducer and activator of transcription \\
TNF & Tumor necrosis factor
\end{tabular}

\section{References}

1. Hawkes, J.E.; Chan, T.C.; Krueger, J.G. Psoriasis pathogenesis and the development of novel targeted immune therapies. J. Allergy Clin. Immunol. 2017, 140, 645-653. [CrossRef] [PubMed]

2. Tollefson, M.M.; Crowson, C.S.; McEvoy, M.T.; Maradit Kremers, H. Incidence of psoriasis in children: A population-based study. J. Am. Acad. Dermatol. 2010, 62, 979-987. [CrossRef]

3. Taylor, S.L.; Petrie, M.; O'Rourke, K.S.; Feldman, S.R. Rheumatologists' recommendations on what to do in the dermatology office to evaluate and manage psoriasis patients' joint symptoms. J. Dermatol. Treat. 2009, 20, 350-353. [CrossRef]

4. Gelfand, J.M.; Neimann, A.L.; Shin, D.B.; Wang, X.; Margolis, D.J.; Troxel, A.B. Risk of myocardial infarction in patients with psoriasis. JAMA 2006, 296, 1735-1741. [CrossRef] [PubMed]

5. Gisondi, P.; Tessari, G.; Conti, A.; Piaserico, S.; Schianchi, S.; Peserico, A.; Giannetti, A.; Girolomoni, G. Prevalence of metabolic syndrome in patients with psoriasis: A hospital-based case-control study. Br. J. Dermatol. 2007, 157, 68-73. [CrossRef]

6. Neimann, A.L.; Shin, D.B.; Wang, X.; Margolis, D.J.; Troxel, A.B.; Gelfand, J.M. Prevalence of cardiovascular risk factors in patients with psoriasis. J. Am. Acad. Dermatol. 2006, 55, 829-835. [CrossRef]

7. Brauchli, Y.B.; Jick, S.S.; Miret, M.; Meier, C.R. Psoriasis and risk of incident cancer: An inception cohort study with a nested case-control analysis. J. Investig. Dermatol. 2009, 129, 2604-2612. [CrossRef] [PubMed]

8. Qureshi, A.A.; Choi, H.K.; Setty, A.R.; Curhan, G.C. Psoriasis and the risk of diabetes and hypertension: A prospective study of US female nurses. Arch. Dermatol. 2009, 145, 379-382. [CrossRef] [PubMed]

9. Piskin, G.; Tursen, U.; Sylva-Steenland, R.M.R.; Bos, J.D.; Teunissen, M.B.M. Clinical improvement in chronic plaque-type psoriasis lesions after narrow-band UVB therapy is accompanied by a decrease in the expression of IFN-gamma inducers-IL-12, IL-18 and IL-23. Exp. Dermatol. 2004, 13, 764-772. [CrossRef] 
10. Piskin, G.; Sylva-Steenland, R.M.; Bos, J.D.; Teunissen, M.B. In vitro and in situ expression of IL-23 by keratinocytes in healthy skin and psoriasis lesions: Enhanced expression in psoriatic skin. J. Immunol. 2006, 176, 1908-1915. [CrossRef]

11. Guttman-Yassky, E.; Lowes, M.A.; Fuentes-Duculan, J.; Zaba, L.C.; Cardinale, I.; Nograles, K.E.; Khatcherian, A.; Novitskaya, I.; Carucci, J.A.; Bergman, R.; et al. Low Expression of the IL-23/Th17 Pathway in Atopic Dermatitis Compared to Psoriasis. J. Immunol. 2008, 181, 7420-7427. [CrossRef] [PubMed]

12. Lee, E.; Trepicchio, W.L.; Oestreicher, J.L.; Pittman, D.; Wang, F.; Chamian, F.; Dhodapkar, M.; Krueger, J.G. Increased Expression of Interleukin 23 p19 and p40 in Lesional Skin of Patients with Psoriasis Vulgaris. J. Exp. Med. 2004, 199, 125-130. [CrossRef] [PubMed]

13. Li, H.; Yao, Q.; Mariscal, A.G.; Wu, X.; Hulse, J.; Pedersen, E.; Helin, K.; Waisman, A.; Vinkel, C.; Thomsen, S.F.; et al. Epigenetic control of IL-23 expression in keratinocytes is important for chronic skin inflammation. Nat. Commun. 2018, 9, 1420. [CrossRef] [PubMed]

14. Oppmann, B.; Lesley, R.; Blom, B.; Timans, J.C.; Xu, Y.; Hunte, B.; Vega, F.; Yu, N.; Wang, J.; Singh, K.; et al. Novel p19 Protein Engages IL-12p40 to Form a Cytokine, IL-23, with Biological Activities Similar as Well as Distinct from IL-12. Immunity 2000, 13, 715-725. [CrossRef]

15. Langrish, C.L.; Chen, Y.; Blumenschein, W.M.; Mattson, J.; Basham, B.; Sedgwick, J.D.; McClanahan, T.; Kastelein, R.A.; Cua, D.J. IL-23 drives a pathogenic T cell population that induces autoimmune inflammation. J. Exp. Med. 2005, 201, 233-240. [CrossRef] [PubMed]

16. Mus, A.M.C.; Cornelissen, F.; Asmawidjaja, P.S.; Van Hamburg, J.P.; Boon, L.; Hendriks, R.W.; Lubberts, E. Interleukin-23 promotes Th17 differentiation by inhibiting T-bet and FoxP3 and is required for elevation of interleukin-22, but not interleukin-21, in autoimmune experimental arthritis. Arthritis Rheum. 2010, 62, 1043-1050. [CrossRef]

17. Warren, R.B.; Blauvelt, A.; Poulin, Y.; Beeck, S.; Kelly, M.; Wu, T.; Geng, Z.; Paul, C. Efficacy and safety of risankizumab vs. secukinumab in patients with moderate-to-severe plaque psoriasis (IMMerge): Results from a phase III, randomized, open-label, efficacy-assessor-blinded clinical trial. Br. J. Dermatol. 2021, 184, 50-59. [CrossRef] [PubMed]

18. Reich, K.; Warren, R.B.; Iversen, L.; Puig, L.; Pau-Charles, I.; Igarashi, A.; Ohtsuki, M.; Falqués, M.; Harmut, M.; Rozzo, S.; et al. Long-term efficacy and safety of tildrakizumab for moderate-to-severe psoriasis: Pooled analyses of two randomized phase III clinical trials (re SURFACE 1 and re SURFACE 2) through 14. Br. J. Dermatol. 2020, 182, 605-617. [CrossRef]

19. Blauvelt, A.; Papp, K.A.; Griffiths, C.E.M.; Randazzo, B.; Wasfi, Y.; Shen, Y.-K.; Li, S.; Kimball, A.B. Efficacy and safety of guselkumab, an anti-interleukin-23 monoclonal antibody, compared with adalimumab for the continuous treatment of patients with moderate to severe psoriasis: Results from the phase III, double-blinded, placebo- and active comparator-. J. Am. Acad. Dermatol. 2017, 76, 405-417. [CrossRef]

20. Langley, R.G.; Tsai, T.F.; Flavin, S.; Song, M.; Randazzo, B.; Wasfi, Y.; Jiang, J.; Li, S.; Puig, L. Efficacy and safety of guselkumab in patients with psoriasis who have an inadequate response to ustekinumab: Results of the randomized, double-blind, phase III NAVIGATE trial. Br. J. Dermatol. 2018, 178, 114-123. [CrossRef]

21. Kulig, P.; Musiol, S.; Freiberger, S.N.; Schreiner, B.; Gyülveszi, G.; Russo, G.; Pantelyushin, S.; Kishihara, K.; Alessandrini, F.; Kündig, T.; et al. IL-12 protects from psoriasiform skin inflammation. Nat. Commun. 2016, 7, 13466. [CrossRef]

22. Ramnath, D.; Tunny, K.; Hohenhaus, D.M.; Pitts, C.M.; Bergot, A.S.; Hogarth, P.M.; Hamilton, J.A.; Kapetanovic, R.; Sturm, R.A.; Scholz, G.M.; et al. TLR3 drives IRF6-dependent IL-23p19 expression and p19/EBI3 heterodimer formation in keratinocytes. Immunol. Cell Biol. 2015, 93, 771-779. [CrossRef]

23. Wang, X.; Wei, Y.; Xiao, H.; Liu, X.; Zhang, Y.; Han, G.; Chen, G.; Hou, C.; Ma, N.; Shen, B.; et al. A novel IL-23p19/Ebi3 (IL-39) cytokine mediates inflammation in Lupus-like mice. Eur. J. Immunol. 2016, 46, 1343-1350. [CrossRef] [PubMed]

24. Wang, X.; Liu, X.; Zhang, Y.; Wang, Z.; Zhu, G.; Han, G.; Chen, G.; Hou, C.; Wang, T.; Ma, N.; et al. Interleukin (IL)-39 [IL-23p19/Epstein-Barr virus-induced 3 (Ebi3)] induces differentiation/expansion of neutrophils in lupus-prone mice. Clin. Exp. Immunol. 2016, 186, 144-156. [CrossRef] [PubMed]

25. Wang, X.; Zhang, Y.; Wang, Z.; Liu, X.; Zhu, G.; Han, G.; Chen, G.; Hou, C.; Wang, T.; Shen, B.; et al. Anti-IL-39 (IL-23p19/Ebi3) polyclonal antibodies ameliorate autoimmune symptoms in lupus-like mice. Mol. Med. Rep. 2017, 17, 1660-1666. [CrossRef] [PubMed]

26. Bridgewood, C.; Alase, A.; Watad, A.; Wittmann, M.; Cuthbert, R.; McGonagle, D. The IL-23p19/EBI3 heterodimeric cytokine termed IL-39 remains a theoretical cytokine in man. Inflamm. Res. 2019, 68, 423-426. [CrossRef] [PubMed]

27. Ecoeur, F.; Weiss, J.; Schleeger, S.; Guntermann, C. Lack of evidence for expression and function of IL-39 in human immune cells. PLoS ONE 2020, 15, e0242329. [CrossRef]

28. Detry, S.; Skladanowska, K.; Vuylsteke, M.; Savvides, S.N.; Bloch, Y. Revisiting the combinatorial potential of cytokine subunits in the IL-12 family. Biochem. Pharm. 2019, 165, 240-248. [CrossRef]

29. Scholz, G.M.; Heath, J.E.; Walsh, K.A.; Reynolds, E.C. MEK-ERK signaling diametrically controls the stimulation of IL-23p19 and

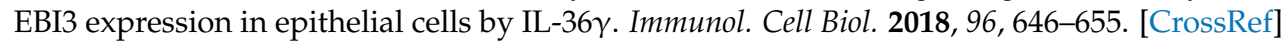

30. Chiricozzi, A.; Guttman-Yassky, E.; Suárez-Fariñas, M.; Nograles, K.E.; Tian, S.; Cardinale, I.; Chimenti, S.; Krueger, J.G. Integrative Responses to IL-17 and TNF- $\alpha$ in Human Keratinocytes Account for Key Inflammatory Pathogenic Circuits in Psoriasis. J. Investig. Dermatol. 2011, 131, 677-687. [CrossRef] 
31. Johansen, C.; Bertelsen, T.; Ljungberg, C.; Mose, M.; Iversen, L. Characterization of TNF- $\alpha-$ and IL-17A-Mediated Synergistic Induction of DEFB4 Gene Expression in Human Keratinocytes through IкBC. J. Investig. Dermatol. 2016, 136, 1608-1616. [CrossRef] [PubMed]

32. Morizane, S.; Nomura, H.; Tachibana, K.; Nakagawa, Y.; Iwatsuki, K. The synergistic activities of the combination of tumour necrosis factor-alpha, interleukin-17A and interferon-gamma in epidermal keratinocytes. Br. J. Dermatol. 2018, 179, 496-498. [CrossRef] [PubMed]

33. Ling, P.; Gately, M.K.; Gubler, U.; Stern, A.S.; Lin, P.; Hollfelder, K.; Su, C.; Pan, Y.C.; Hakimi, J. Human IL-12 p40 homodimer binds to the IL-12 receptor but does not mediate biologic activity. J. Immunol. 1995, 154, 116-127.

34. Shimozato, O.; Sato, A.; Kawamura, K.; Chiyo, M.; Ma, G.; Li, Q.; Tagawa, M. The secreted form of p28 subunit of interleukin (IL)-27 inhibits biological functions of IL-27 and suppresses anti-allogeneic immune responses. Immunology 2009, 128, e816-e825. [CrossRef]

35. Stumhofer, J.S.; Tait, E.D.; Iii, W.J.Q.; Hosken, N.; Spudy, B.; Goenka, R.; Fielding, C.A.; O'Hara, A.C.; Chen, Y.; Jones, M.L.; et al. A role for IL-27p28 as an antagonist of gp130-mediated signaling. Nat. Immunol. 2010, 11, 1119-1126. [CrossRef] [PubMed]

36. Maaser, C.; Egan, L.J.; Birkenbach, M.P.; Eckmann, L.; Kagnoff, M.F. Expression of Epstein-Barr virus-induced gene 3 and other interleukin-12-related molecules by human intestinal epithelium. Immunology 2004, 112, 437-445. [CrossRef]

37. Devergne, O.; Hummel, M.; Koeppen, H.; Le Beau, M.M.; Nathanson, E.C.; Kieff, E.; Birkenbach, M. A novel interleukin-12 p40-related protein induced by latent Epstein-Barr virus infection in B lymphocytes. J. Virol. 1996, 70, 1143-1153. [CrossRef] [PubMed]

38. Kudo, H.; Wang, Z.; Jinnin, M.; Nakayama, W.; Inoue, K.; Honda, N.; Nakashima, T.; Kajihara, I.; Makino, K.; Makino, T.; et al. EBI3 Downregulation Contributes to Type I Collagen Overexpression in Scleroderma Skin. J. Immunol. 2015, 195, 3565-3573. [CrossRef]

39. Gerosa, F.; Baldani-Guerra, B.; Lyakh, L.A.; Batoni, G.; Esin, S.; Winkler-Pickett, R.T.; Consolaro, M.R.; De Marchi, M.; Giachino, D.; Robbiano, A.; et al. Differential regulation of interleukin 12 and interleukin 23 production in human dendritic cells. J. Exp. Med. 2008, 205, 1447-1461. [CrossRef]

40. Martínez-Barricarte, R.; Markle, J.G.; Ma, C.S.; Deenick, E.K.; Ramírez-Alejo, N.; Mele, F.; Latorre, D.; Mahdaviani, S.A.; Aytekin, C.; Mansouri, D.; et al. Human IFN- $\gamma$ immunity to mycobacteria is governed by both IL-12 and IL-23. Sci. Immunol. 2018, 3. [CrossRef] [PubMed]

41. Ehst, B.; Wang, Z.; Leitenberger, J.; McClanahan, D.; De La Torre, R.; Sawka, E.; Ortega-Loayza, A.G.; Strunck, J.; Greiling, T.; Simpson, E.; et al. Synergistic induction of IL-23 by TNF $\alpha$, IL-17A, and EGF in keratinocytes. Cytokine 2021, $138,155357$. [CrossRef] [PubMed]

42. Bai, F.; Li, G.G.; Liu, Q.; Niu, X.; Li, R.; Ma, H. Short-Term Efficacy and Safety of IL-17, IL-12/23, and IL-23 Inhibitors Brodalumab, Secukinumab, Ixekizumab, Ustekinumab, Guselkumab, Tildrakizumab, and Risankizumab for the Treatment of Moderate to Severe Plaque Psoriasis: A Systematic Review and Network Meta-Analysis of Randomized Controlled Trials. J. Immunol. Res. 2019, 2019, 2546161. [CrossRef] [PubMed] 